\title{
SURVEYING BEST SuITABLE SCHEDULING ALGORITHM FOR WIMAX- WI-FI INTEGRATED HETEROGENEOUS NETWORK
}

\author{
Poulomi Das ${ }^{1}$, Siladitya Sen $^{2}$, Arindam Banerjee $^{3}$ \\ ${ }^{1,2,3}$ Electronics \& Communication Engineering Department, Heritage Institute \\ of Technology, Kolkata, West Bengal, India \\ ${ }^{1}$ das.poulomi214@gmail.com \\ ${ }^{2}$ Siladitya.sen@heritageit.edu \\ ${ }^{3}$ arindam. banerjee.mtece130heritageit.edu.in
}

\begin{abstract}
To provide uninterrupted service to all subscribers in a wireless network, we need to incorporate a low cost, flexible Heterogeneous network which will be able to link with any kind of network for efficient spectrum utilization, hence improved system capacity. In this connection, Wi-Fi/ Wi MAX integrated network seems to be an ideal solution as it is able to provide easy deployment, high speed data rate and wide range coverage with high throughput, low end to end delay, flat and low jitter. Wi-Fi/ WiMAX integrated network provides Quality of Service $(Q o S)$ that can support all kinds of real-time application in wireless networks that includes priority scheduling and queuing for bandwidth allocation that is based on traffic scheduling algorithms within wireless networks. In this paper, we have designed a Wi-Fi/ WiMAX integrated network and analyze the performance of different scheduling algorithms for that integrated network and highlight our findings on the scheduling algorithm which will give the best performance for a heterogeneous network.
\end{abstract}

\section{KEYWORDS}

Wi-Fi, WiMAX, QoS, Scheduling

\section{INTRODUCTION}

Chronologically, high speed internet access from cable, Digital Subscriber Line (DSL), and other fixed broad-band connections are going to be replaced by wireless hotspots, Wi-Fi \& WiMAX services. Due to the advent of portable, low cost \& user friendly devices, users are attracted towards wireless services. As time \& users demand uninterrupted services, so the last mile winner WiMAX will be able to cover large areas in metropolitan, suburban and rural terrain with high speed mobile broadband internet access called wide area networks (WANs), where Wi-Fi can provide high data rate in short range communication. Providing proper network services to every user, from time of registration to time to leave is the main function of a network. As both networks support mobility so integration of both networks can be the best solution to maintain proper QoS. A network can be accessed at a time by various types of users for different applications. Managing those large numbers of traffic can be done by the scheduling algorithm. The scheduling algorithm map users with various service classes \& add priority for source or sync. Demand for proper scheduling algorithm increases proportionally with number of subscribers \& numbers of various applications in wireless communication. In a heterogeneous

Rupak Bhattacharyya et al. (Eds) : ACER 2013,

pp. 329-346, 2013. (C) CS \& IT-CSCP 2013

DOI : $10.5121 /$ csit.2013.3231 
network there is priority not only for users but also for different access points \& base stations have assigned different priority \& the scheduling of both variable-size real-time and non-realtime connections is not standardised. So still this is an open field of research and development. In this paper, we have proposed a survey on scheduling schemes used in WiMAX \& Wi-Fi networks for both uplink and downlink traffic. Also this paper will enlighten the best suitable scheduling algorithm for an integrated heterogeneous network to maintain proper QoS for voice, data, real time \& non real time applications.

\section{WiMAX ARCHITECTURE}

The basic WiMAX (IEEE 802.16) architecture consists of one Base Station (BS) and one or more Subscriber Station (SS). BS acts as a central entity to transfer all the data from SSs through two basic operational modes: mesh and point-to multipoint (PMP). Meanwhile, transmissions take place through two independent channels: Downlink Channel (from BS to SS) and Uplink Channel (from SS to BS). The Uplink Channel is shared among all SSs, while the Downlink Channel is used only by BS [1]. In the PMP mode, the SSs are only allowed to communicate through the BS. While in the mesh mode, subscriber stations (SS) can communicate with each other as well as with the base station (BS).

In 1999 the IEEE Standards Board established a new working group called IEEE 802.16 [2]. The purpose of this working group was to develop and publish air interface standards for wireless metropolitan area networks. In 2003 the WiMAX Forum was established in order to promote and enable the deployment of a new broadband access technology based on the 802.16 standards, called WiMAX [3]. The first WiMAX system was based on IEEE 802.16-2004 standard [2]. This standard offers fixed broadband wireless communications using roof top mounted Customer Premises Equipment. In December, 2005 the IEEE completed the 802.16e-2005 amendment, which added new features to support mobile applications. This resulting standard is now commonly known by the WiMAX Forum as mobile WiMAX, release 1.0 [4]. Based on the mandatory features and some of the optional features needed for enhanced mobility and QoS support of the 802.16e-2005 standard, the WiMAX Forum released the first Mobile WiMAX, release 1.0, in late 2006 .

At present when WiMAX Forum is released WiMAX 2.0. This release is based on the IEEE $802.16 \mathrm{~m}$ standard, see [5], and will improve spectrum efficiency, latency and scalability to achieve wider bandwidths in challenging environments. According to [3], release 2.0 supports following features:

2.1 Multimedia session continuity. This provides the ability to maintain continuity of multimedia sessions during handovers.

2.2 3 GPP/2 interworking (optimized handover). Is a handover improvement in order to switch to $3 \mathrm{GPP} / 2$ networks and vice versa.

2.3 Network management, including self-organized/optimized networks (SONs). This functionality provides the ability to (automatic) increase the overall network performance, quality and reliability.

2.4 Seamless Wi-Fi-WiMAX handover. Wi-Fi-WiMAX handover is a mechanism used to support a seamless handover from Wi-Fi to WiMAX and vice versa.

2.5 Roaming enhancements. This is an improvement of roaming, within the mobile WiMAX release 1.0. 
2.6 Support for multi-hop relay stations. This is a mechanism that enables the use of multihop relay stations, which extend or enhance the coverage of a base station.

2.7 Support for femto-cells. This is an ability to use cell phone connectivity around house by using WiMAX.

2.8 Device reported metrics. These are mechanisms that monitor devices.

The co-existence of different network users should be achieved with relatively low control overhead. For these reasons, the frame format, the subcarrier mapping schemes and the pilot structure are being modified for $802.16 \mathrm{~m}$ with respect to $802.16 \mathrm{e}$. Each IEEE $802.16 \mathrm{~m}$ frame consists of a downlink (DL) and an uplink (UL) part separated in time by an OFDMA symbol and is of variable size [6,7]. The (downlink or uplink) frame begins by control information that all users employ to synchronize and to determine if and when they should receive or transmit in the given frame. Control information is followed by data transmission by the base station (in the downlink sub-frame) or the mobile stations (in the uplink sub-frame). The general frame structure is illustrated in Figure 1. Data is organized into a hierarchy of Super-frames, Frames, sub-frames, and OFDM symbols. Super-frames last $20 \mathrm{~ms}$ and contain four $5 \mathrm{~ms}$ frames, each of which contains 8 sub-frames unless the channel size is $7 \mathrm{MHZ}$ in which case frames contain 6 subframes, or 8.75 MHZ, in which case frames contain 7 sub-frames [SDD]. Sub-frames fall into one of four categories, three of which are employed as part of $802.16 \mathrm{~m}$ and a fourth that is included for legacy operation with $802.16-2009$ devices operating on $8.75 \mathrm{MHz}$ channels. These types are:

Type $1-6$ OFDMA symbols

Type $2-7$ OFDMA symbols

Type $3-5$ OFDMA symbols

Type 4 - 9 OFDMA symbols [8]

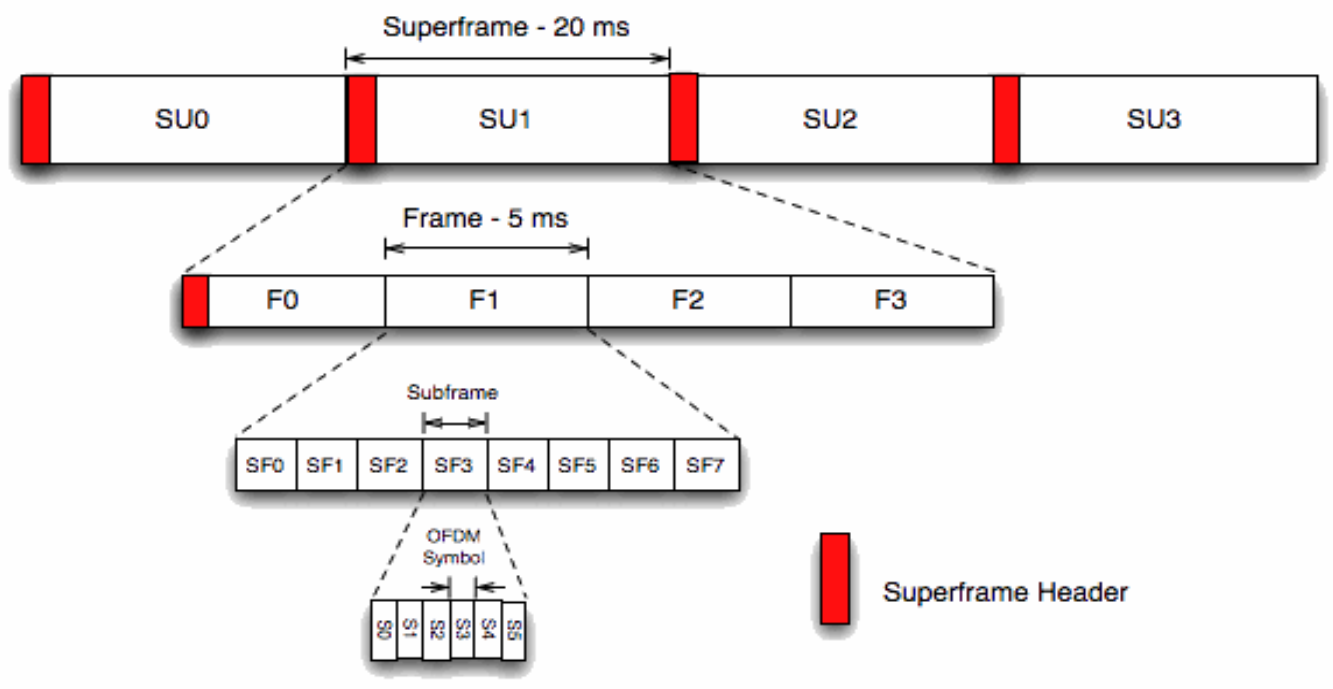

Figure1. Frame Structure for WiMAX (IEEE 802.16m)

\subsection{16m MAC}

The Media Access Control (MAC) layer is responsible for encapsulating data and feeding it into the PHY layer for transmission, as well as receiving new data that is verified then passed on to 
higher layers. Note that higher layers are largely independent of the network they run on and therefore are not dealt with in $802.16 \mathrm{~m}$ [8].

\subsection{HARQ}

HARQ, or Hybrid Automatic Repeat Request is a system used by $802.16 \mathrm{~m}$ to ensure all packets are transmitted and correctly received. While there are several variations of ARQ, 802.16m uses one primarily based on stop-and-wait. This means that when each frame is sent, the sender waits until it received an ACK (acknowledgement) before sending the next frame. Multiple HARQ channels can run in parallel (up to 16), mitigating the performance hit of waiting for an ACK before sending more data. Each of these channels has a unique identifier that is determined differently for UL and DL traffic. For DL traffic, it is simply the HARQ Channel ID (ACID). For UL traffic this identifier is a combination of the ACID and the index of the sub-frame containing the HARQ data. Legacy systems used a different version of HARQ, which is supported in $802.16 \mathrm{~m}$ as a special case. The old version is called Chase Combining, and involves retransmitting exactly the same data in the event that an ACK is not received [Bacioccola10]. $802.16 \mathrm{~m}$ uses a variant of HARQ known as Incremental Redundancy (IR). IR retransmits the same data with a (potentially) different encoding. The idea behind using different coding is that if there is some interference preventing the data from being received correctly, an encoding with more redundancy or simply a different bit-pattern may either work better, or different portions may be received allowing the incorrectly received data to be combined to create a correct copy. Chase Combining is simply the special case where the new encoding is the same as the old one $[8,9]$.

\subsection{QoS}

The MAC layer of an $802.16 \mathrm{~m}$ network is based on the concept of connections, which are conceptualized as unidirectional data flows (each of which will generally be paired with a data flow in the opposite direction). Each flow is assigned a four-bit Flow ID (FID). The FID can be combined with a 12-bit Station ID (STID) to generate a network-unique 16-bit identifier for that flow. The separation of FIDs and STIDs is useful due to the fact that in Handovers the FIDs do not need to change. This allows all connections to be very quickly re-established by simply changing the STID to the new value assigned by the new ABS. There is a downside in that the legacy model allowed many more than the 16 connections per MS limit dictated by the four bit FID. Legacy systems used the full 16-bit connection ID for each connection, which allowed up to 216 connections per station. However, each of these connection IDs had to be reassigned on handover, which created significant overhead. Each flow in $802.16 \mathrm{~m}$ may have QoS service parameters [Bacioccola10], which are negotiated between the ABS and AMS when the flow is setup. These parameters are the same as the parameters assigned to connections in legacy systems. An important part of QoS is bandwidth allocation. The bandwidth request protocol has been reworked for $802.16 \mathrm{~m}$. In legacy systems, a five-message request was needed, which specified the bandwidth grant side explicitly each time. In $802.16 \mathrm{~m}$ there is a shorter 3-message grant request available that will automatically assume some default size and allow two messages to be skipped, thereby lowering latency $[9,10]$. 


\subsection{Handoff Time Requirement}

Table1. Handover Downtime $[8,10]$

\begin{tabular}{|l|c|c|c|}
\hline Handover Type & $\begin{array}{c}\text { Intra frequency } \\
\text { Handoff }\end{array}$ & \multicolumn{2}{|c|}{ Inter frequency Hand off } \\
\cline { 2 - 4 } & & Within Spectrum Band & $\begin{array}{c}\text { Between Spectrum } \\
\text { Band }\end{array}$ \\
\hline Handover Time(ms) & 27.5 & 40 & 60 \\
\hline
\end{tabular}

All of these changes resulted in significant improvements to performance, with a throughput gain of at least $2 \mathrm{x}$ and a decrease in latency in all cases.

\section{OVERVIEW OF WI-FI NETWORK}

The new wireless LAN standard IEEE 802.11n—ratified as „WLAN Enhancements for Higher Throughput" in September 2009-features a number of technical developments that promise up to six-times the performance in wireless LANs.

The new technology offers the following advantages:

\subsection{Higher effective data throughput}

The 802.11n standard includes a number of new mechanisms to significantly increase available bandwidth. Current wireless LAN standards based on $802.11 \mathrm{a} / \mathrm{g}$ enable physical data rates (gross data rates) of up to $54 \mathrm{Mbps}$, which turn out to be approx. $22 \mathrm{Mbps}$ net. Networks based on 802.11 currently achieve a gross data throughput of up to $300 \mathrm{Mbps}$ (in reality approx. 120 to $130 \mathrm{Mbps}$ net) - theoretically the standard defines up to $600 \mathrm{Mbps}$ with four data streams. For the first time speeds can actually exceed the $100 \mathrm{Mbps}$ of cable-based Fast Ethernet networks, which are currently standard in most workplaces.

\subsection{Improved and more reliable wireless coverage}

The new 802.11n technologies not only increase data throughput, but they also reduce areas without reception at the same time. This results in better signal coverage and improved stability for significantly better utilization of wireless networks, in particular for users in professional environments.

\subsection{Greater range}

Data throughput generally decreases when the distance between receiver and transmitter increases. The overall improved data throughput allows wireless LANs based on 802.11n to achieve greater ranges, as a significantly stronger wireless signal is received by the Access Point a given distance than in $802.11 \mathrm{a} / \mathrm{b} / \mathrm{g}$ networks.

\subsection{Improved OFDM modulation}

Like 802.11a/g, 802.11n uses the OFDM scheme (Orthogonal Frequency Division Multiplex) as its method of modulation. This modulates the data signal not on just one carrier signal but in parallel over several. The data throughput that can be achieved with OFDM modulation depends on the following parameters, among other things:

- Number of carrier signals: Whereas $802.11 \mathrm{a} / \mathrm{g}$ uses 48 carrier signals, $802.11 \mathrm{n}$ can use a maximum of 52 . 
- Payload data rate: Airborne data transmission is fundamentally unreliable. Even small glitches in the WLAN system can result in errors in data transmission. Check sums are used to compensate for these errors, but these take up a part of the available bandwidth. The payload data rate indicates the ratio between theoretically available bandwidth and actual payload. $802.11 \mathrm{a} / \mathrm{g}$ can operate at payload rates of $1 / 2$ or $3 / 4$ while $802.11 \mathrm{n}$ can use up to $5 / 6$ of the theoretically available bandwidth for payload data.

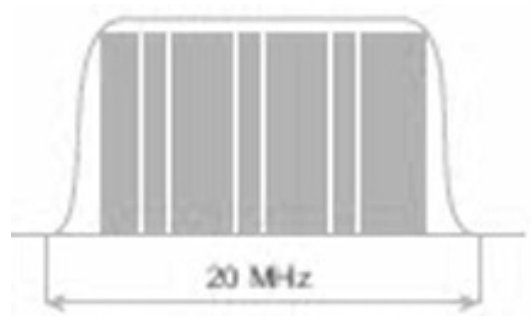

Figure2. IEEE 802.11n: 52 carrier signals

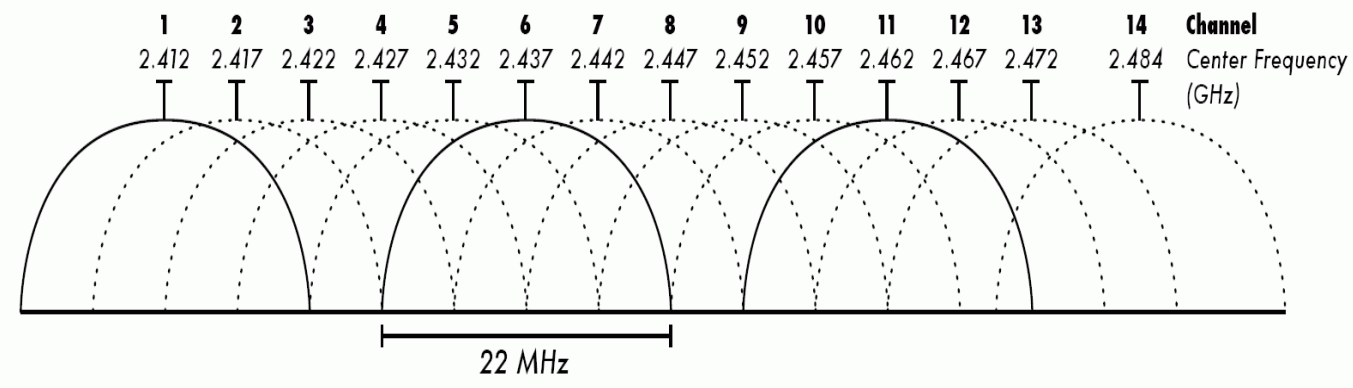

Figure 3. Channel Frequency

The $2.4000-2.4835 \mathrm{GHz}$ band is divided into thirteen channels, each with a width of $22 \mathrm{MHz}$ but spaced only $5 \mathrm{MHz}$ apart. Channel 1 has a centre frequency of $2.412 \mathrm{GHz}$ and channel 13 is centred on $2.472 \mathrm{GHz}$. To avoid channels overlapping, therefore, they need to be spaced as shown above (for example channels 1, 6 and 11 may be used within the same coverage area without interfering with each other).

Each IEEE 802.11 frame has a header, a variable length payload, and a Frame Check Sequence (FCS). Frames may be control frames, data frames, or management frames. The frame is preceded by a preamble and a Physical Layer Convergence Protocol (PLCP) header, as shown below. 


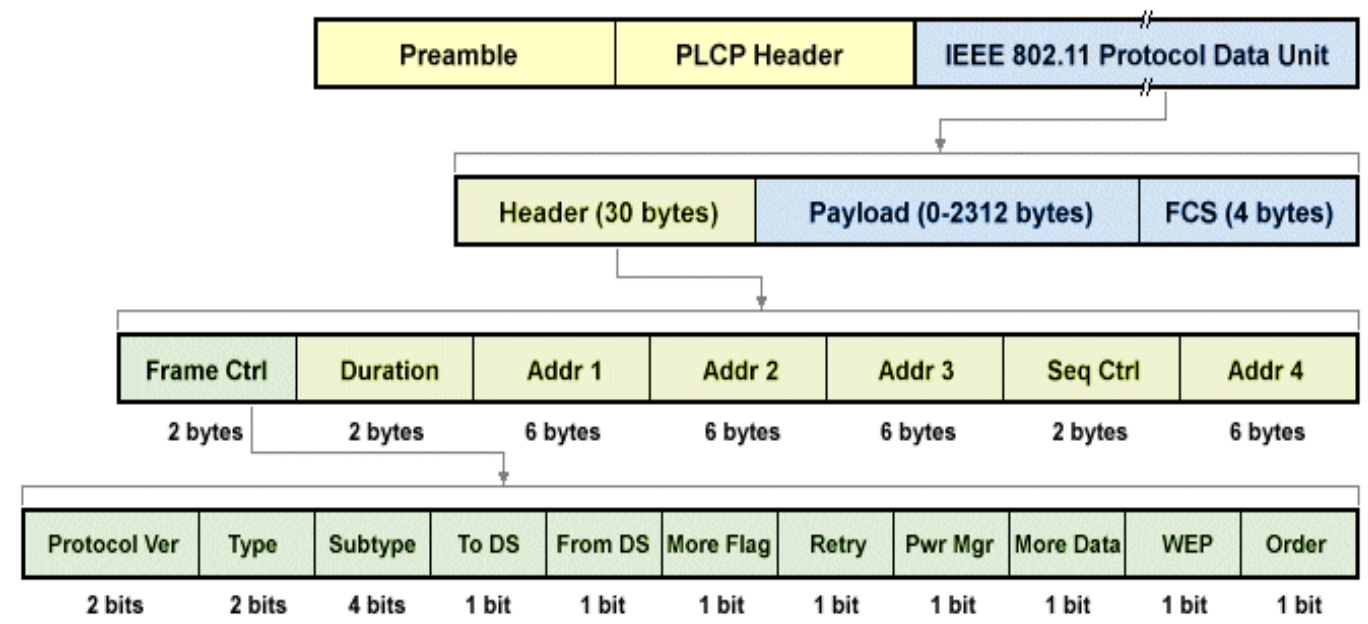

Figure 4. IEEE 802.11 Frame Format

The first two bytes of the IEEE 802.11 header are taken up by the frame control field, which consists of a number of sub-fields that contain information about the frame, such as the protocol version, frame type, whether power management is active, and so on. The address fields carry the MAC address of the source and destination devices, as well as that of one or more access points. The sequence control field is used for ordering message fragments, and for the identification of any duplicated frames. The variable-length payload can carry user data or control and management information, depending on the frame type. The Frame Check Sequence occupies the last 4 bytes of the frame, and is used for error detection purposes.

\subsection{Control frames}

- Acknowledgement (ACK) - upon receiving a data frame (and assuming no errors are detected), the destination device sends an acknowledgement to the source device. If the source device does not receive an acknowledgement within a predetermined period of time, it will retransmit the frame.

- Request to Send (RTS) - as part of an optional collision avoidance scheme, a wireless device wishing to transmit data may send a Request to Send frame before sending the data itself, and will wait for the destination device to reply with a Clear to Send frame.

- Clear to Send (CTS) - sent in response to a Request to Send frame to indicate that it is OK for the device that sent the RTS frame to transmit. The Clear to Send frame includes a time value to indicate to other stations the time period during which they should refrain from transmitting.

\subsection{Management frames}

- Authentication - the network adapter on a wireless device sends an authentication frame to the access point to identify itself. For open system authentication, the access point responds with its own authentication frame indicating whether or not it accepts the sender's identity. For shared key authentication, the authentication frame sent by the access point contains challenge text, which the wireless device must encrypt with the correct encryption key and send back to the access point in a further authentication frame. The access point will be able to determine whether the challenge text has been correctly 
encrypted using its own decryption key, and responds with a final authentication frame signifying whether or not it accepts the sender's identity.

- Association request - the network adapter on a wireless device sends an association request frame to an access point to establish an association. Once an association is established, the access point can synchronise with the sending device and allocate resources to it. The request frame carries information such as the data rates supported by the sender, and the SSID of the network it wishes to associate with. If the access point accepts the request, it reserves memory space and establishes an association ID for the wireless adapter.

- Association response - sent by an access point to a wireless device in response to an association request frame. If the response contains an acceptance of the association request, it will also specify the association ID and information about supported data rates.

- Beacon - broadcast at regular intervals by an access point to advertise its presence to any wireless devices within range. The frame includes the SSID for the access point.

- De authentication - sent by one station to another to terminate secure communications.

- Disassociation - sent one station to another to terminate an association. If sent by a wireless device to an access point, the access point can de-allocate any memory reserved for the device and delete its association ID.

- Probe request - sent by a station to elicit information (for example, to determine what other stations are in range).

- Probe response - sent in response to a probe request frame, this frame includes information such as supported data rates etc.

- Re association request - used by a roaming wireless device to request a handover when it detects an access point with a stronger signal than the one with which it is currently associated.

- Re association response - sent by an access point in response to a reassociation request frame. If the response contains an acceptance of the association request, it will also specify the association ID and information about supported data rates.

Table 2: IEEE 802.11 Specifications

\begin{tabular}{|c|c|c|c|c|}
\hline $\begin{array}{c}\text { Standard } \\
\text { Approved }\end{array}$ & JEEE 802.11a & IEEE 802.11b & IEEE 802.11g & IEEE 802.11n \\
\hline $\begin{array}{c}\text { Maximum } \\
\text { Data Rate }\end{array}$ & $54 \mathrm{Mbps}$ & $11 \mathrm{Mbps}$ & $54 \mathrm{Mbps}$ & Jot yet ratified \\
\hline Modulation & OFDM & DSSS or CCK & $\begin{array}{c}\text { DSSS or CCK } \\
\text { or OFDM }\end{array}$ & $\begin{array}{c}\text { DSSS or CCK or } \\
\text { OFDM }\end{array}$ \\
\hline RF Band & $5 \mathrm{GHz}$ & $2.4 \mathrm{GHz}$ & $2.4 \mathrm{GHz}$ & $2.4 \mathrm{GHz}$ or 5GHz \\
\hline $\begin{array}{c}\text { Number of } \\
\text { Spatial } \\
\text { Streams }\end{array}$ & 1 & 1 & 1 & $1,2,3$ or 4 \\
\hline Channel Width & $20 \mathrm{MHz}$ & $20 \mathrm{MHz}$ & $20 \mathrm{MHz}$ & $\begin{array}{c}20 \mathrm{MHz} \text { or } \\
40 \mathrm{MHz}\end{array}$ \\
\hline
\end{tabular}

\section{QOS IN WI-FI NETWORK}

The basic WLAN is an important NGN access network but has least developed QoS mechanisms, due to its contention based medium access mechanisms [11]. In addition, the existing WLAN QoS schemes implement QoS on individual networks independently. Since IEEE 802.11 is connectionless, a host that sends a transmission cannot detect the state of the network or the state 
of the destination before transmission. The MAC layer defines two different access methods, the 'listen before transmission' or distributed coordination function (DCF) which is used for random access 'best-effort' traffic with several other algorithms specific to the WLAN. The point coordination function (PCF) uses contention free period (CFP). The IEEE 802.11 standard specifies that the basic technique DCF is mandatory and other procedures CSMA/CA with RTS/CTS and Hybrid coordination function (HCF) are optional [12]. HCF is more popular as it uses both by assigning different waiting periods to node with different real-time data transmission constraints. CFP is the HCF period and contention period (CP) is the duration of contention in Fig. 4 below. Beacons or control frames are used to start super-frames in CFP. Traffic on these network technologies, however, is subject to delay, making bandwidth availability and delivery time difficult to predict. Although high-priority traffic typically arrives at its destination before lower priority traffic as per demands of time-flexible telecom applications, it cannot be guaranteed to arrive within a specified time needed in time critical applications. Despite the fact that QoS is more difficult to implement on connectionless networks, there is growing interest in developing QoS for IP-based networks [13].

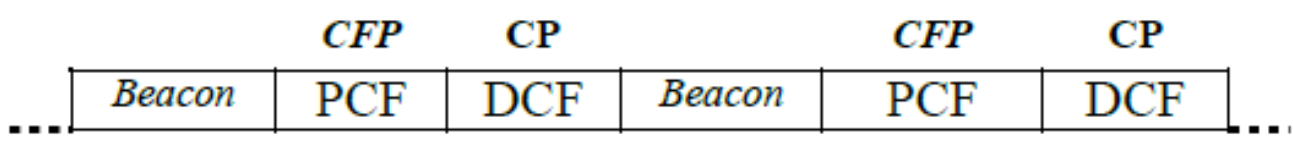

Figure 5. 802.11 Super-frame format with CFP and CP periods

In connectionless networks, the two ends are not aware of the traffic being sent on the channel, for example User datagram protocol (UDP) in the transport layer which is easier to implement but not reliable as it maintain no state information and packet losses. On the other hand, Transmission control protocol (TCP) ensures reliable data transfer with error checking and reporting at both ends.

In 802.11e there are two co-ordination functions known as Hybrid Coordination Function (HCF). The first coordination function is the Enhanced Distributed Coordination function (EDCF), which is a QoS enabled version of DCF and the HCF Controlled Channel Access (HCCA), which is similar to the PCF [11]. In 802.11e, combined EDCF and HCF controlled channel access mechanisms are implemented for ensuring QoS in WLANs.

\section{WIMAX QUALITY OF SERVICES}

WiMAX standard defines 5 service classes to support its wide range of applications as endorsed by IEEE 802.16 .

\subsection{Unsolicited grant services (UGS)}

This class of service is designed to support fixed-sized data packets at a constant bit rate (CBR) such as E1/T1 lines that can sustain real-time data stream applications. This service provides guaranteed throughput, latency and jitter to the necessary levels as TDM services. UGS is used mainly to support Constant Bit Rate (CBR) services found in voice applications such as voice over IP $[14,15,16,17,18]$ 


\subsection{Real-time Polling Services (rtPS)}

This class of service is designed to support real-time service flow that generates variable-sized data packets on a periodic interval with a guaranteed minimum rate and guaranteed delay. The mandatory service flow parameters that define this service are inclusive of minimum reserved traffic rate, maximum sustained traffic rate, maximum latency and request/transmission policy. rtPS is used extensively in MPEG video conferencing and streaming [14, 15, 16, 17, 18].

\subsection{Non-real-time Polling Service (nrtPS)}

This class of service is designed for non-real-time traffic with no delay guaranteed. The delay tolerant data stream consists of variable-sized data packets. The applications provided by this service are time-insensitive and a minimum amount of bandwidth. This service is especially suitable for critical data application such as in File Transfer Protocol (FTP) [14, 15, 16, 17, 18].

\subsection{Extended real-time Polling Service (ertPS)}

This class of service provides real-time applications which generate variable-sized data packets periodically that require guaranteed data rate and delay with silence suppression. This service is only defined in IEEE 802.16e- 2005. During the silent periods, no traffic is sent and no bandwidth is allocated. However, there is a need to have a BS poll during the MS to determine the end of the silent periods. ertPS is featured in VoIP with silence suppression [14, 15, 16,17, 18].

\subsection{Best-Effort Services (BE)}

This class of service provides support for data streams whereby no minimum service-level guarantee is required. The mandatory service flow parameters that define this service include maximum sustained traffic rate, traffic priority and request/transmission policy. BE supports data streams found in Hypertext Transport Protocol (HTTP) and electronic mail (e-mail) [14, 15, 16, $17,18]$.

\section{SCHEduling AlgorithmS}

The main focus of this research study is to examine the scheduling schemes in WiMAX network. In order to specify high network performance, an efficient scheduling algorithm is essential as it manages and controls the provision of an efficient level of QoS support. Although many scheduling algorithms have been proposed in the literature for WiMAX network, the design of the algorithms are challenged by having to support different levels of services, fairness and implementation complexity. Many researchers have compared their proposal schemes on different scheduling schemes, but there is no common, simple and standardized packet scheduling to make their comparisons with. In this study, six carefully selected scheduling algorithms in WiMAX wireless network are investigated. These algorithms which are considered the most dominant and popular include Diffserv-Enabled (Diffserv), Round-Robin (RR), Self-Clocked-Fair (SCF), Strict-Priority (SP), Weighted-Fair Queuing (WFQ) and Weighted Round Robin (WRR). Furthermore, these common packet scheduling schemes provides QoS support for real time applications in IEEE 802.16 system.

\subsection{Diffserv-Enabled}

Diff-serv is a simple, scalable and measurable mechanism for classifying and managing network traffic. Besides, it provides low-latency with guaranteed service to critical network traffic as well 
as to non-critical services. It relies on the principle of traffic classification by involving the 6-bit Differentiated Services Code Point (DSCP) field in the header of IP packets to classify the packet and indicate the per-hop behaviour (PHB). DSCP replaces the out dated IP precedence in classifying and prioritizing types of traffic. Every router on the Diff-serv network is configured to differentiate traffic based on class so that each traffic class can be managed differently, ensuring preferential treatment for higher priority traffic on the network [19].

\subsection{Round-Robin (RR)}

It is designed for a time-sharing system whereby the scheduler assigns time slots to each queue in equal portions without priority. It starts with the highest priority queue with packets, services a single packet, then visits the next lower priority queue with packets, and continues servicing every single packet from each queue. This is carried on until each queue with packets has been serviced once. Every queue is allocated with the same portion of system resources regardless of the channel condition, ultimately utilizing the same resources. However, the RR scheduler has the same bandwidth efficiency as a random scheduler, so it cannot guarantee different QoS requirements for each queue [20,21].

\subsection{Self-Clocked-Fair (SCF)}

It is an efficient queuing scheme which satisfies the quality of services (QoS) in broadband implementation. The algorithm is based on the concept of virtual time that adopts the concept of an internally generated virtual time as the index of work in progress. It links virtual time to the work progress in the fluid-flow fair queuing (FFQ). As virtual time function is involved in determining the order of which packet should be served next, the virtual time that is produced depends very much on the progress of work in the actual packet based queuing system. This scheme is efficient for the internal generation of virtual time as it involves negligible overhead. This is because virtual time is easily computed from the packet situated at the head of the queue. In addition, the SCFQ algorithm can accomplish easier implementation and it can maintain the fairness attribute in virtual time function. [22, 23, 24].

\subsection{Strict-Priority (SP)}

In Strict-Priority algorithm, the selection order is based on the priority of weight order. The packets are first categorized by the scheduler depending on the quality of service (QoS) classes and then allocated into different priority queues. The algorithm services the highest priority queue until it is empty, after which, it moves to the next highest priority queue. Thus, strict-priority algorithm may not be suitable in WiMAX network. This is because there is no compensation for inadequate bandwidth. Also this technique is only appropriate for low-bandwidth serial lines that currently uses static configuration which does not automatically adapt to changing network requirements. Finally, this process may result in bandwidth starvation for the low priority QoS classes whereby the packets may not even get forwarded and no guarantee is offered to one flow [19].

\subsection{Weighted-Fair-Queuing (WFQ)}

This algorithm is employed for uplink traffic in WiMAX with different size packets. As it caters to different size packets, it emphasizes on providing fair scheduling for the different flows by assigning finish times to the packets. The finish times are based on the size and weight of the packets. In general, the WFQ algorithm outperforms the WRR due to variable size packets. 
However, the weaknesses of WFQ algorithm are, the start time of a packet is not taken into consideration, and it can lower the scheduler system if many packets occur in the priority region $[25,26]$.

\subsection{Weighted Round Robin (WRR)}

It is a scheduling algorithm implemented for resource sharing in a computer or network. In fact, WRR is an extension of the Round Robin (RR) algorithm. In a network, WRR serves a number of packets that are computed by normalizing weight of data divided by the average of packet size from nonempty connection queue. It begins by classifying packets into a variety of service classes followed by assigning a queue that is determined by the different percentage of bandwidth. Finally, it is serviced in round robin order. Since the bandwidth is assigned according to the weights, the algorithm will not provide good performance in the presence of variable size packets. However, WRR method makes certain that all service classes have access to at least some configured amount of network band width to avoid bandwidth starvation [14, 25, 27].

\section{Heterogeneous Network ArChitecture}

Here we designed a two layer hierarchy model for better services. The network schematic architectures are shown below:
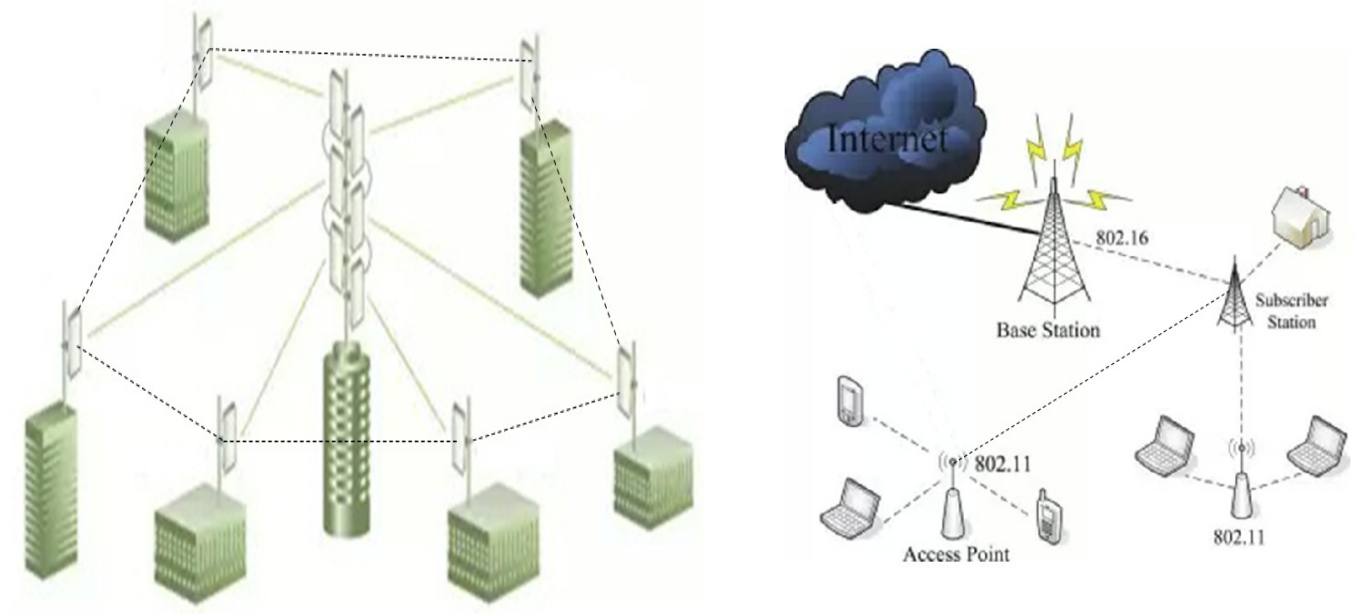

Figure 6. Schematic Diagram of Heterogeneous WiMAX-Wi-Fi Network

In our designed network all outer networks are connected by WiMAX base stations. All access points of IEEE 802.11 are connected with coupling device situated on roof top or in prime location. If any user comes out side of buildings the call will hand off to nearby WiMAX station. 


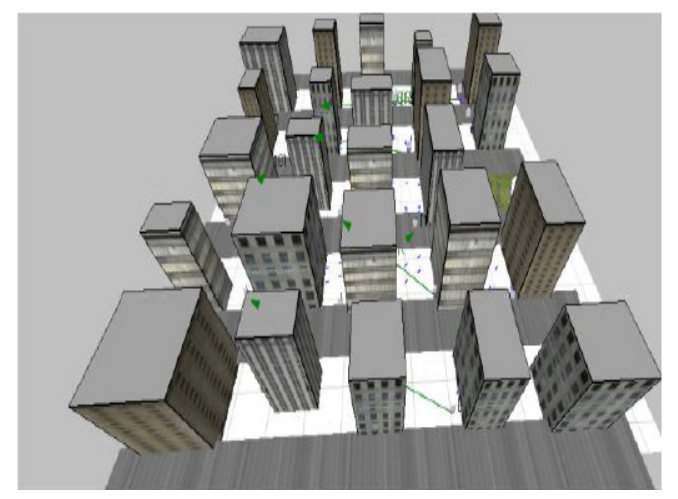

Figure 7. WiMAX-Wi-Fi Network Designing in QualNet Simulator

Using QualNet simulator we have designed a scenario for metropolitan city. There is WiMAX base station (SS) on every roof top which are connected with a central base station. These Base Stations are connected with Wi-Fi access point. Subscribers are connected with access points. There is hand off between Wi-Fi to Wi-Fi network, Wi-Fi to WiMAX network \& WiMAX to WiMAX network. This heterogeneous network model packet transfers from Wi-Fi to WiMAX network or vice versa. In this model there are 50 mobile stations are in scenario. We check one after one scheduling algorithms. Finally all algorithms simultaneously for 10 mobile station each.

\section{ANALYSiS OF THE RESUlT}

In this paper, we have analyzed the throughput, average end to end delay, average jitter for Wi-Fi \& WiMAX integrated network. For this analysis we have considered two cases. In the first case, a comparison is going on among different scheduling algorithms for specific amount of load in WiFi \& WiMAX integrated network From the first analysis we can conclude which will give the best performance for the heterogeneous network. Form Figure 8,10,12 we can conclude that WRR scheduling algorithm gives the best performance for heterogeneous network in terms of maximum throughput, minimum jitter and end to end delay. In second case, for best scheduling algorithm that is WRR, we have analyzed the performance of the network in terms of throughput, end to end delay and average jitter ( in Figure 9,11,13) considering four different types of scenarios which are given below.

$>$ In first case, connections established between users under Wi-Fi coverage with users direct under WiMAX coverage.

$>$ In second case, connections are established between the users under same Wi-Fi coverage area.

$>$ In third case connections are established between users under Wi-Fi network to users under another Wi-Fi network. These two Wi-Fi networks are under different WiMAX network.

$>$ In fourth case connections are established between two mobile Wi-Fi networks under different WiMAX network. 


\subsection{Throughput}

In communication network, throughput or network through-put is the average rate of successful message delivery over a communication channel. Data may be delivered over a physical or logical link, measured in bits per second. The system throughput or average throughput is the sum of the data rates that are delivered to all the terminals in a network [28].

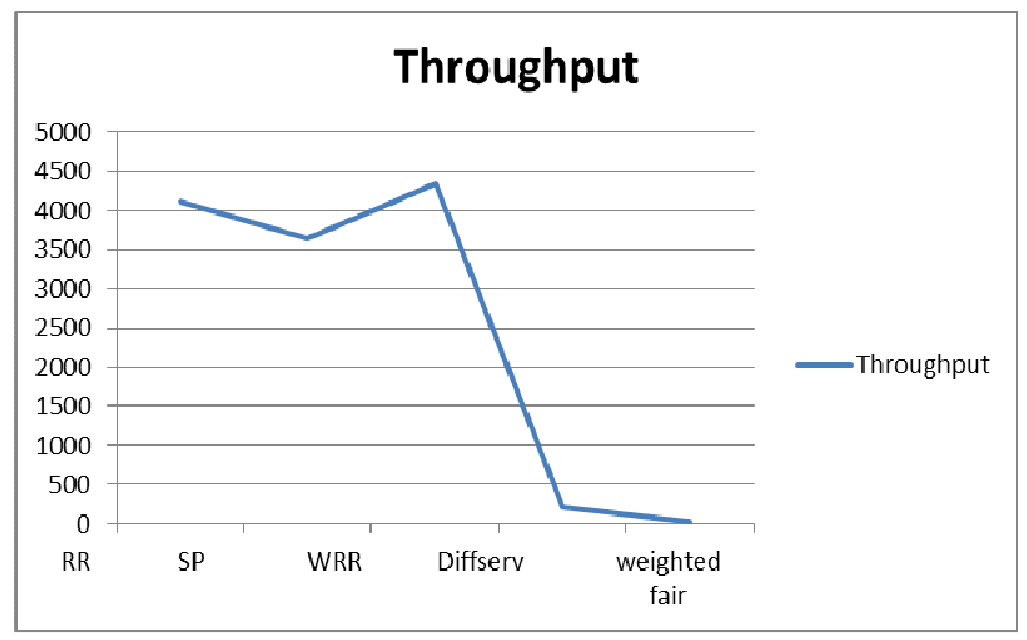

Figure 8: Throughput for different scheduling algoriths (10 stations each)

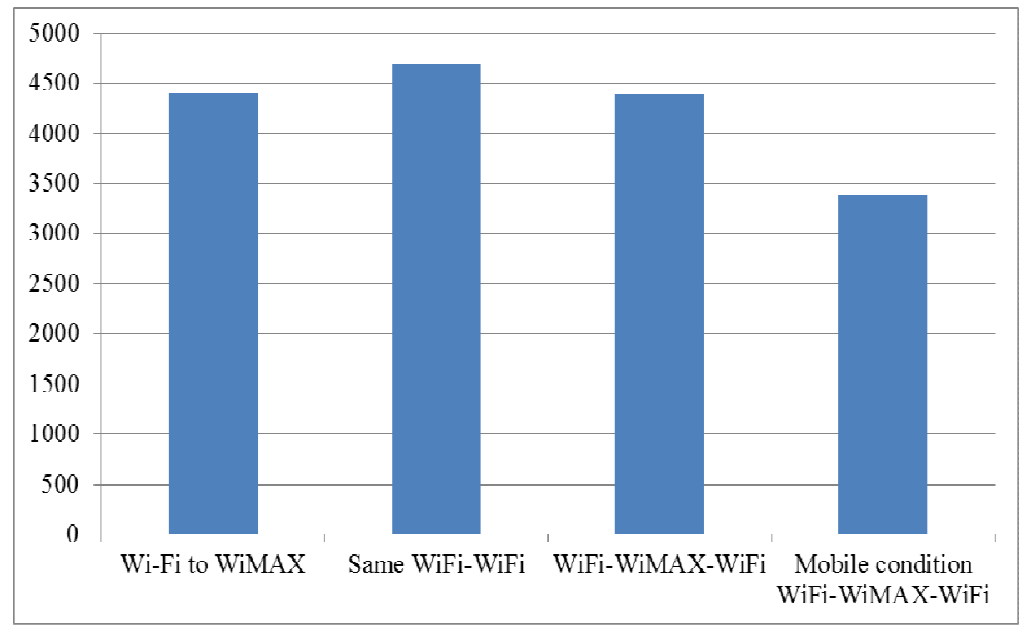

Figure 9: Throughput of heterogeneous network for four different scenarios using WRR Algorithm

\subsection{Jitter}

As the packets transmit from source to destination will reach the destination with different delays. A packet's delay varies with its position in the queues of the routers along the path between source and destination and this position can vary unpredictably. This variation in delay is known as Jitter. The jitter increases at switches along the path of a connection due to many factors, such as conflicts with other packets wishing to use the same links, and nondeterministic propagation delay in the data-link layer. Jitter can seriously affect the quality of streaming audio and/or video. 
A network could possibly have zero Jitter. Jitter for respective precedence bits are calculated and compared. [29]

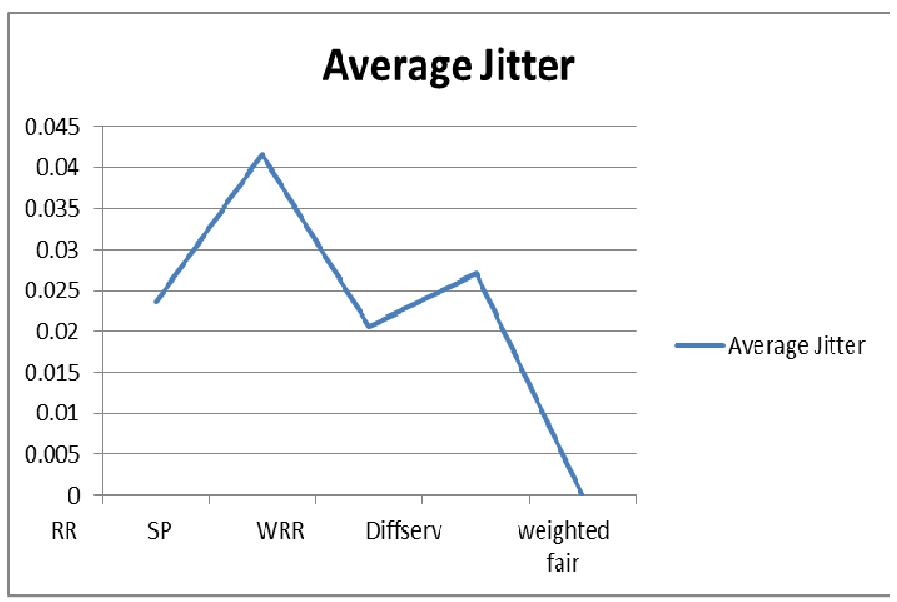

Figure 10: Jitter for different scheduling algoriths (10 stations each)

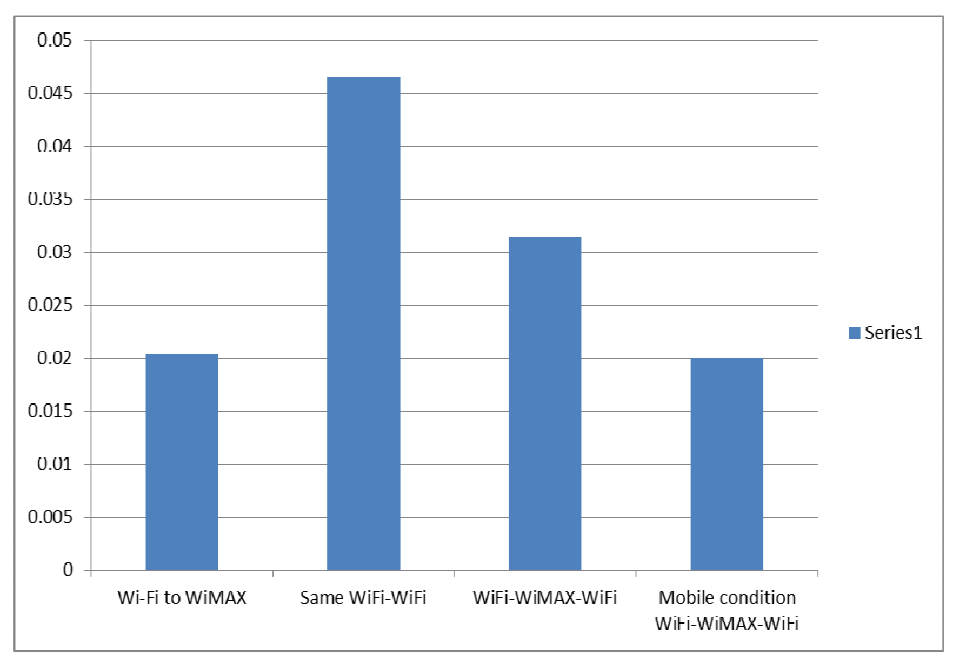

Figure 11: Jitter of heterogeneous network for four different scenarios using WRR Algorithm

\subsection{End-to-End Delay}

Due to queuing and different routing paths, a data packet may take a longer time to reach its destination .The end-to-end delay experienced by the packets for each flow the individual packet delay are summed and the average is computed. [30]

dend-end $=\mathrm{N}[$ dtrans+dprop+dproc $][30]$

Where dend-end is end-to-end delay, dtrans is transmission delay, dprop is propagation delay, dproc is processing delay and $\mathrm{N}$ is number of links. 


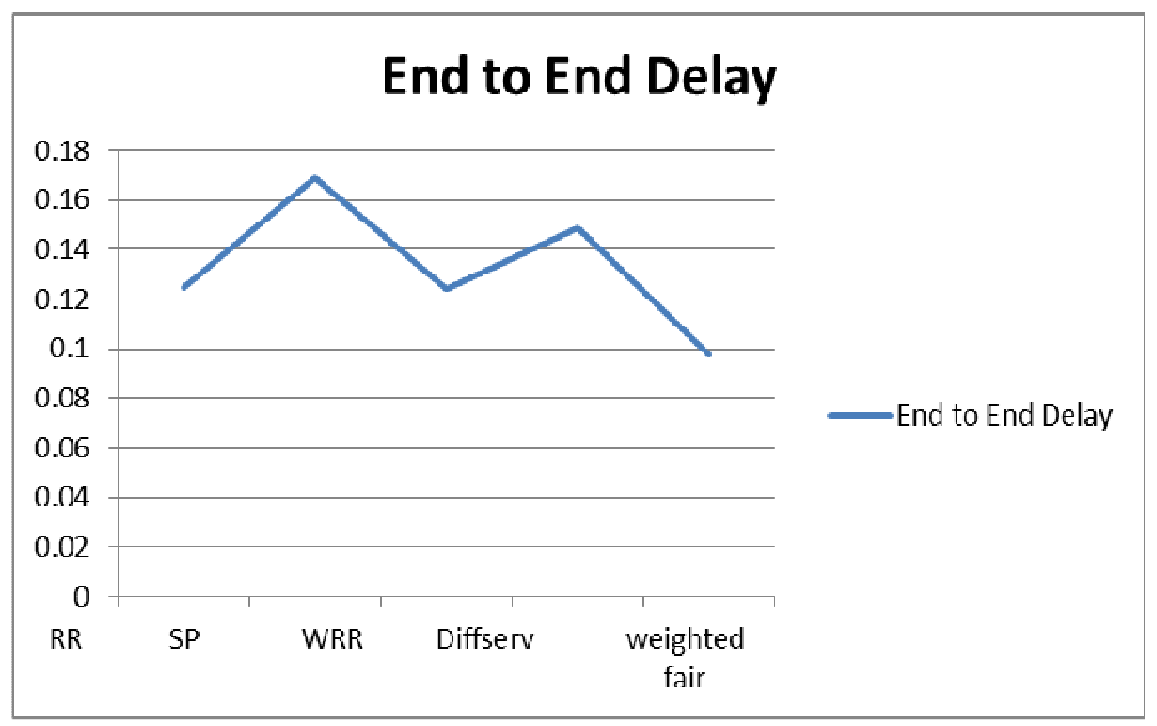

Figure 12: End to End Delay for different scheduling algoriths (10 stations each)

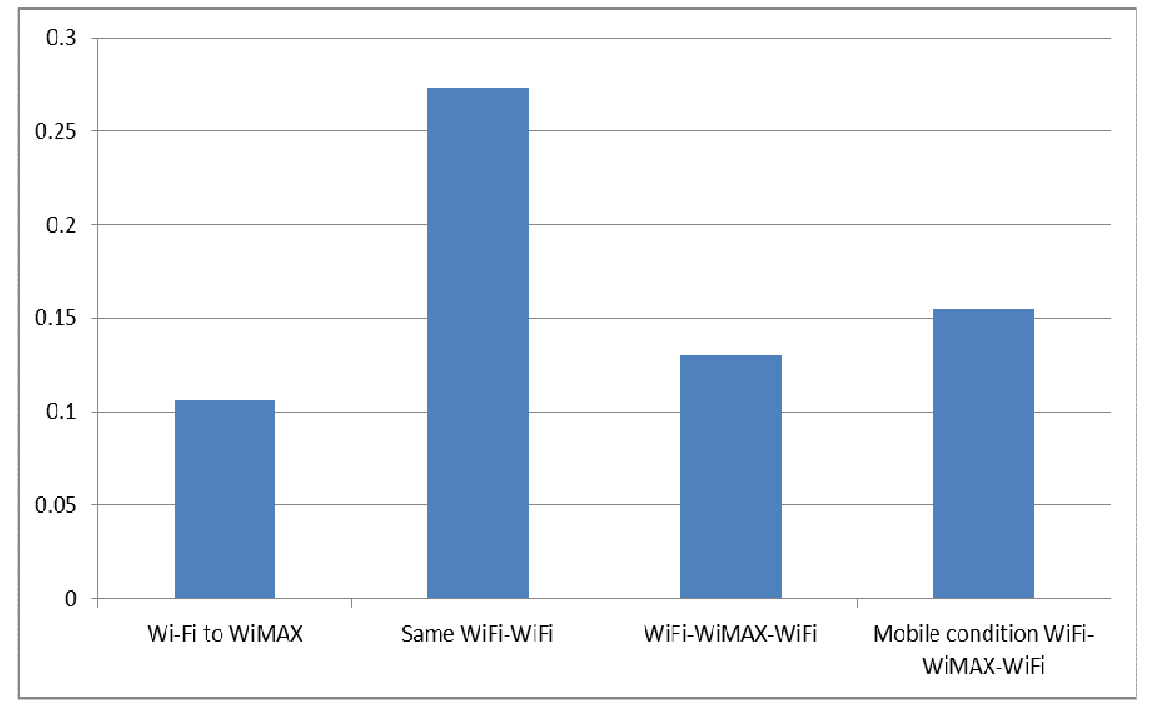

Figure 13: End to End Delay of heterogeneous network for four different scenarios using WRR Algorithm

\section{Conclusions}

From the above results we can conclude that Heterogeneous model will be able to improve the capacity of a wireless network with efficient utilization of network resources. Through the use of QualNet simulation we have concluded that WRR provides best performance for mixed network. We have also verified the performance of the network for various user conditions under WRR scheduling algorithm. There is a scope to improve performance of the network using the same Wi-Fi access point without accessing the WiMAX base station.. 


\section{REFERENCES}

[1] Jun Wang, Weijia Jia, and Liusheng Huang, "An Efficient Centralized Scheduling Algorithm for IEEE 802.16 Multi-radio Mesh Networks", ICUIMC '2008 Proceedings of the 2nd international conference on Ubiquitous information management and communication, Suwon, Korea, January 31 February 01, 2008.

[2] "IEEE Standard for Local and Metropolitan Area Networks Part 16: Air Interface for Fixed broadband

[3] Wireless Access Systems,” IEEE Std 802.16-2004 (Revision of IEEE Std 802.16-2001), pp. 0_1-857, 2004.

[4] K. Etemad, "Overview of mobile WiMAX technology and evolution,"IEEE Communications Magazine, vol. 46, no. 10, pp. 31-40, 2008.

[5] M. Tran, G. Zaggoulos, A. Nix et al., "Mobile WiMAX: Performance Analysis and Comparison with Experimental Results," Vehicular Technology Conference, 2008. VTC 2008-Fall. IEEE 68th, pp. 15, 2008.

[6] G. A. Prasath, F. Cheng Peng, and M. Maode, "QoS scheduling for group mobility in WiMAX," Communication Systems, 2008. ICCS 2008. $11^{\text {th }}$ IEEE Singapore International Conference on, pp. 1663-1667, 2008.

[7] Koon Hoo Teo, Zhifeng Tao, Jinyun Zhang,"The Mobile Broadband WiMAX standard[Standards in Nutshell]”, Signal Processing Magazine, IEEE, p144-148, 2007.

[8] Etemad, K., "Overview of mobile WiMAX technology and evolution", Communications magazine, IEEE, vol. 46, p31-40,2008.

[9] Srinivasan, Roshni (ed), Hamiti, Shkumbin (ed), "IEEE 802.16m System Description Document (SDD)," IEEE 802.16 Task Group m, September 2009, http://www.ieee802.org/16/tgm/docs/80216m09_0034r2.zip

[10] Bacioccola10] A. Bacioccola, C. Cicconetti, C. Eklund, L. Lenzini, Z. Li, E. Mingozzi, "IEEE 802.16: History, Status and Future Trends", Computer Communications, Volume 33, Issue 2, 15 February 2010, Pages 113-123, http://www.sciencedirect.com/science/article/B6TYP-4XNF43R1/2/f0a4cc67a688adb985355e0ea97d642f

[11] Yaghoobi, Hassan, "Mobile WiMAX Update and IEEE 802.16m," IEEE, March 2009, http://ieeetmc.net/r6/scv/sps/WiMAX_Update_802-16m.pdf

[12] Anis KOUBAA and Mario Alves, "A Two-Tiered Architecture for Real-Time Communications in Large-Scale Wireless Sensor Networks: Research Challenges”, ECRTS'05, Spain, 2005.

[13] Mario Marchese, QoS Over Heterogeneous Networks, Wiley, 07.

[14] F. Agharebparast and V.C.M Leung, "QoS support in the UMTS/GPRS backbone network using DiffServ", Global Telecommunications Conference, 2002. GLOBECOM 02. IEEE 17-21 Nov. 2002, Vol. 2, pp: 1440- 1444.

[15] Jani Lakkakorpi, Alexander Sayenko and Jani Moilanen. (2008). Comparison of Different Scheduling Algorithms for WiMAX Base Station Deficit Round-Robin vs. Proportional Fair vs. Weighted Deficit Round-Robin. Proceedings

[16] Chakchai So-In, Raj Jain, and Abdel-Karim Tamimi. (2009). Scheduling in IEEE 802.16e Mobile WiMAX Networks: Key Issues and a Survey. IEEE Journal on selected areas in communications, vol. 27(2). February. pp. 156- 171.

[17] Mikael Gidlund and Gang Wang. (2009). Uplink Scheduling Algorithms for QoS Support in Broadband Wireless Access Networks. Journal of communications. Vol. 4(2). pp. 133-142.

[18] Ashish Jain and Anil K. Verma. (2008). Comparative Study of Scheduling Algorithms for WiMAX. Proceedings of the National Conference on Mobile and Pervasive Computing, Compc 08, 7-8 August. Chennai, India. pp. 10-13.

[19] Ahmed H. Rashwan, Hesham M. ElBadawy and Hazem H. Ali. (2009). Comparative Assessments for Different WiMAX Scheduling Algorithms. Proceedings of the World Congress on Engineering and Computer Science WCECS 2009. Vol. I. 20-22 October, 2009. San Francisco, USA. pp. 362-366.

[20] Yuxiao Jia, Miguel Lopez Guerrero, Ognian Kabranov, Dimitrios Makrakis and Luis Orozco Barbosa. (2003). Design and testbed implementation of adaptive MPLS- Diffserv enabled virtual private networks. Proceedings of the IEEE Canadian Conference Electrical and Computer Engineering, CCECE 2003. 4-7 May. Montreal, Canada. pp 965-968. 
[21] Jason Nieh, Chris Vaill and Hua Zhong. (2001). Virtual-Time Round-Robin:An O(1) Proportional Share Scheduler. Proceedings of the USENIX Annual Technical Conference. 25-30 June. Boston, Massachusetts, USA. pp. 245-259.

[22] Jason Nieh, Chris Vaill and Hua Zhong. (2001). Virtual-Time Round-Robin:An O(1) Proportional Share Scheduler. Proceedings of the USENIX Annual Technical Conference. 25-30 June. Boston, Massachusetts, USA. pp. 245-259.

[23] S. Jamaloddin Golestani. (1994). A Self-Clocked Fair Queueing Scheme for Broadband Applications. Proceedings of the IEEE Conference on Computer Communications, 13th Annual Joint Conference of the IEEE Computer and Communications Societies, INFOCOM '94, 12-16 June. Toronto, Ontario, Canada. pp. 636-646.

[24] Hassan Halabian, Hossein Saidi and Reyhaneh Changiz. (2008). LVT-SCFQ: A Modified Self Clocked Fair Queueing Algorithm for Broadband Networks. Proceedings of the 3rd International Conference on Broadband Communications, Information Technology \& Biomedical Applications BroadCom 2008. 23- 26 November. Pretoria, Gauteng, South Africa. pp. 175-180.

[25] Hassan Halabian, Hossein Saidi and Reyhaneh Changiz. (2008). LVT-SCFQ: A Modified Self Clocked Fair Queueing Algorithm for Broadband Networks. Proceedings of the 3rd International Conference on Broadband Communications, Information Technology \& Biomedical Applications BroadCom 2008. 23- 26 November. Pretoria, Gauteng, South Africa. pp. 175-180.

[26] Ahmed H. Rashwan, Hesham M. ElBadawy and Hazem H. Ali. (2009). Comparative Assessments for Different WiMAX Scheduling Algorithms. Proceedings of the World Congress on Engineering and Computer Science WCECS 2009. Vol. I. 20-22 October, 2009. San Francisco, USA. pp. 362-366.

[27] Richard Kautz, Raymond Keh, Kee Chaing Chua and Alberto Leon-Garcia. (2000). A Distributed Fair Queuing (DFQ) Architecture for Wireless ATM Local Access Networks. International journal of wireless information networks. vol. 7(4). pp. 221-229.

[28] Najah Abu Ali, Pratik Dhrona and Hossam Hassanein. (2009). A performance study of uplink scheduling algorithms in pointto- multipoint WiMAX networks. Computer communications. vol. 32. pp. 511-521.

[29] S Chatterjee, S Sen, S De "Effects on Throughput for Different Data rates of IEEE 802.11b Standard on Wi-Fi Network using QualNet Simulator" National Conference on Materials, Devices and Circuits in Communication Technology (MDCCT 2012)

[30] Jitter Control in QoS Networks" Yishay Mansour and Boaz Patt-Shami

[31] Lea Skorin-Kapov, Darko Huljenic, Dario Mikic, Danko Vilendecic, "Analysis of End-to-End QoS for Networked Virtual Reality Services in UMTS" IEEE Communications Magazine, April 2004 .

\section{AUTHORS}

Poulomi Das is a M. Tech final year student of Heritage Institute of Technology, Kolkata. She had worked on micro-strip antenna designing \& at present working on Wireless Communication Network Designing under guidance of Prof. Siladitya Sen.

Prof. Siladitya Sen, Associate Professor in Heritage Institute of technology Kolkata, Alumni of Jadavpur University, West Bengal.

Arindam Banerjee is a M. Tech (ECE) final year student of Heritage Institute of Technology, Kolkata. He had worked on Optimised Sensor Network \& Fuzzy Logic. At present working on Wireless Communication Network Designing under guidance of Prof. Siladitya Sen.

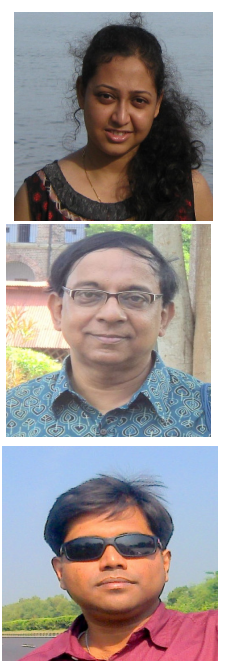

